\title{
Prácticas de crianza asociadas al comportamiento negativista desafiante y de agresión infantil
}

\author{
Parental Rearing Practices Associated to Oppositional Defiant \\ Disorder and Children Aggressive Behavior \\ Práticas de criação associadas ao comportamento negativista \\ desafiante e de agressão infantil
}

\author{
Silvia Morales Chainé, Violeta Félix Romero, Marcela Rosas Peña, \\ Faribia López Cervantes, Javier Nieto Gutiérrez* \\ Universidad Nacional Autónoma de México
}

Doi: dx.doi.org/10.12804/apl33.01.2015.05

\section{Resumen}

Las prácticas de crianza de los padres se asocian al comportamiento problemático infantil que predice el inicio temprano del comportamiento delictivo y del consumo de drogas en la adolescencia. El objetivo de este trabajo es describir los estilos de crianza relacionados con el comportamiento negativista desafiante o agresivo en niños, por medio de un estudio descriptivo correlacional. Se evaluaron 300 participantes, con un promedio de 34 años de edad de 18 entidades de la República mexicana, seleccionados por muestreo, por cuota de instituciones de salud pública que asistieron voluntariamente al programa de crianza positiva por algún problema de conducta con alguno de sus hijos, que estaban entre los 2 y los 12 años de edad. Se utilizaron los auto-reportes y un sistema de observación directa del comportamiento parental en situaciones simuladas de interacción. Los resultados indicaron que el porcentaje de niños con comportamiento negativista desafiante fue mayor que el de niños con conducta agresiva. El reporte y el comportamiento de los padres de dar instrucciones claras, establecimiento de reglas, solución de problemas e interacción social positiva fueron estilos de crianza que se relacionaron confiablemente con un reporte de menor conducta negativista desafiante y de agresión infantil. Palabras clave: Crianza positiva, conducta infantil, negativismo desafiante, agresión

\section{Abstract}

Parental rearing practices are associated to children's problem behavior, which predicts early onset criminal behavior and drug use in adolescents. The goal of the

* Silvia Morales Chainé, Facultad de Psicología, Universidad Nacional Autónoma de México; Violeta Félix Romero, Facultad de Psicología, Universidad Nacional Autónoma de México; Marcela Rosas Peña, Facultad de Psicología, Universidad Nacional Autónoma de México; Faribia López Cervantes, Facultad de Psicología, Universidad Nacional Autónoma de México; Javier Nieto Gutiérrez, Facultad de Psicología, Universidad Nacional Autónoma de México.

La correspondencia relacionada con este artículo debe ser dirigida a Silvia Morales Chainé, Facultad de Psicología, UNAM. Av. Universidad 3004. Col. UNAM, CU. Coyoacán 04510. México. Distrito Federal. Correo electrónico: smchaine@gmail.com

Para citar este artículo: Morales, C. S., Félix, R. V., Rosas, P. M., López, C. F., Nieto, G. J. (2015). Prácticas de Crianza Asociadas al Comportamiento Negativista Desafiante y de Agresión Infantil. Avances en Psicología Latinoamericana 33(1), 57-76. doi: dx.doi.org/10.12804/apl33.01.2015.05 
paper was to describe the parental rearing practices related to the oppositional defiant disorder and to aggressive behavior by means of a correlational study. We assessed 300 participants, from a non random sample, with an average age of 34 years, from 18 states of Mexico, selected from public health institutions, who voluntarily participated in the positive rearing program because of behavior problems of their children, aged 2 to 12 years. We used self-reports and an observational system of the parents' behavior in interactional simulated situations. The results showed that the percentage of children with oppositional defiant disorder was greater. Giving clear instructions, establishing rules, problem solving and positive social interaction, established by parents' self-reporting and behavior observation, were parental rearing styles reliably associated with less reports of oppositional defiant and aggressive behavior.

Keywords: Positive child rearing practices, children behavior, oppositional defiant, aggression

\section{Resumo}

As práticas de criação dos pais se associam ao comportamento problemático infantil que prediz o início prematuro do comportamento delitivo e de consumo de drogas na adolescência. $\mathrm{O}$ objetivo deste trabalho foi descrever os estilos de criação relacionados com o comportamento negativista desafiante ou agressivo em crianças por meio de um estudo descritivo correlacional. Avaliaram-se 300 participantes com uma idade média de 34 anos de 18 entidades da república mexicana selecionados por amostragem por participação de instituições de saúde pública que assistiram voluntariamente ao programa de criação positiva por algum problema de conduta com algum dos seus filhos entre os 2 e os 12 anos de idade. Utilizaram-se os auto-relatos e um sistema de observação direta do comportamento parental em situações simuladas de interação. Os resultados indicaram que a porcentagem de crianças com comportamento negativista desafiante foi maior que a porcentagem das crianças com conduta agressiva. O reporte e o comportamento dos pais de dar instruções claras, estabelecimento de regras, solução de problemas e interação social positiva foram estilos de criação que se relacionaram confiavelmente com um reporte de menor conduta negativista desafiante e de agressão infantil.

Palavras-chave: Criação positiva, conduta infantil, negativismo desafiante, agressão

En México existe interés por estudiar los problemas de conducta infantil, los factores de riesgo asociados, así como el desarrollo de procedimientos exitosos para la reducción efectiva del comportamiento antisocial en la infancia (Morales \& Vázquez, 2011). Los niños que despliegan conducta antisocial tienden a mostrar agresión severa y crónica, así como conducta delictiva en etapas posteriores del desarrollo (Frick, Stickle, Dandreaux, Farrell \& Kimonis, 2005). Es decir, la alta ocurrencia de problemas de conducta en los niños tiene relación con su alta probabilidad de progresión hacia diversos escenarios como la escuela y la comunidad (Ayala, Pedroza, Morales, Chaparro \& Barragán, 2002; Fajardo \& Hernández, 2008) y hacia etapas posteriores del desarrollo como la adolescencia y la adultez (Clark, Vanyukov \& Cornelius, 2002; Foster, Brennan, Biglan, Wang \& Al-Ghaith, 2002; Loeber et al., 2005).

Entre el $4 \%$ y el $12 \%$ de la población mundial infantil presenta problemática conductual (Baker \& Abbott-Feinfield, 2007). En México el $6.1 \%$ de la población ha presentado problemas de conducta alguna vez en su vida (Medina-Mora et al., 2003). Los problemas más frecuentes en niños entre los 4 y 7 años de edad han sido la conducta negativista desafiante, la desobediencia, la conducta agresiva y la hiperactividad. Se estima que el comportamiento negativista desafiante afecta a entre un $2 \%$ y $16 \%$ de la población infantil del mundo (American Psychiatric Association, 2005), mientras que en México el 1.5\% de la población infantil lo padece.

De acuerdo con el DSM-V (American Psychiatric Association, APA, 2013), el comportamiento negativista desafiante es un patrón recurrente de 
conductas de berrinche, discusión con adultos, oposición, desobediencia, irritabilidad, enojo y resentimiento hacia las figuras de autoridad y que provoca deterioro en la actividad social, escolar o familiar. Cornell y Frick (2007) señalan la relevancia de observar un bajo nivel de culpa por el incumplimiento de normas y un bajo de nivel de empatía con las figuras de autoridad en niños que muestran comportamientos característicos de este trastorno. Por su parte, Patterson (1982) ha definido específicamente a la agresión como la coerción que utilizan los individuos para influenciar o controlar las conductas de los demás (Ayala et al., 2002). Así, el comportamiento del orden negativista desafiante puede progresar y transformarse en comportamiento antisocial (pegar, escupir, culpar a otros), o característicos de la violación de normas sociales (romper reglas, escaparse, consumir drogas) y hasta de la violación de propiedad privada — robar, mentir y cometer actos delictivos en grupo (Barkley, 1997; Frick \& White, 2008)—. Por lo que resulta importante prevenir la progresión de los problemas de conducta infantil desarrollando estrategias de reducción exitosas del comportamiento delictivo en la adolescencia.

La probabilidad de que un individuo se comporte agresivamente aumenta si incrementa la presencia de factores de riesgo que superan a sus protectores. Ayala et al. (2002) evaluaron los factores de riesgo, protectores y la generalización del comportamiento agresivo, por medio de un estudio longitudinal de tres años, con una muestra de 345 niños mexicanos en edad escolar, así como la predicción de estos comportamientos a otros escenarios. Utilizaron registros observacionales de interacción del niño con los padres, maestros y otros niños e instrumentos psicométricos de evaluación. Los autores señalaron que los factores asociados con el comportamiento antisocial en la infancia pueden clasificarse en cuatro grupos: (a) los relacionados con las características del niño (temperamento, irritabilidad, disposiciones genéticas, problemas neurológicos, la impulsividad y la baja capacidad intelectual); (b) aquellos relacionados con las características de los padres — altos niveles de estrés, problemas de salud física, psicológica o emocionalidad negativa; (Bradley et al., 2003)—; (c) los que se refieren a la pobre interacción familiar y deficientes prácticas de crianza (Patterson, 1982; Scahill et al., 2006) incluyendo la negligencia (Dadds, Maujean \& Fraser, 2003) o la falta de monitoreo y supervisión (Ayala et al., 2002); y (d) los involucrados con el contexto social en el que se insertan las familias (determinantes familiares, comunitarios, sociales o históricos). También reportaron la generalización del comportamiento agresivo del hogar hacia escenarios escolares y por medio del tiempo. Sin embargo, afirmaron que no se conocen los procesos del desarrollo que se asocian con el mantenimiento del comportamiento antisocial en niños (Frick \& Marsee, 2006).

A partir de la identificación de los factores de riesgo, la interacción entre padres e hijos ha llamado la atención y centrado el interés, particularmente, en el estudio de las prácticas de crianza que predicen el comportamiento antisocial. Concretamente, Ayala et al. (2002) encontraron que la disciplina irritable explosiva, darle instrucciones inespecíficas al niño y agredirlo, así como los altos niveles de estrés por su auto-percepción en la dificultad para desempeñar su papel de padres y de pobre competencia, determinaron el comportamiento agresivo de los niños. Dentro de los factores protectores, se encontró que una organización familiar adecuada, fijar metas de vida, la religiosidad, la cohesión, la disciplina flexible y congruente que no depende del estado de ánimo de los padres y la supervisión de las actividades del niño, se asociaron con su desarrollo social y familiar.

Particularmente, con el fin de describir detalladamente qué prácticas de crianza pueden promover o prevenir el desarrollo o mantenimiento de problemas de conducta en niños, Cornell y Frick (2007) trabajaron con 87 díadas madre-hijo, de clase media, caucásicas, con niños entre los 3 y 5 años de edad. Los niños eran denominados 
por sus maestros y padres como conductualmente inhibidos (controlados) o desinhibidos (hasta impulsivos) y mostraron distintos niveles de culpa por incumplimiento de reglas y empatía con las figuras de autoridad (referentes a que los comportamientos observables se relacionaban con ambas categorías). Analizaron los efectos de interacción entre el negativismo o desafío infantil y los estilos parentales con su nivel de asociación con comportamientos pertenecientes a las categorías del estudio: culpa y empatía infantil. Los resultados indicaron que las prácticas del estilo autocrático orientadas a establecer la obediencia infantil promovieron que los niños con comportamiento desafiante o de reto a la autoridad internalizaran las normas de los padres generando un mayor porcentaje de comportamiento pro-social en ellos. Esto significa que, para el caso de los niños que presentan el trastorno negativista desafiante, caracterizado por conducta externalizada desinhibida y con bajos índices de comportamiento característico de culpa o empatía con las figuras de autoridad, la disciplina basada en el establecimiento claro y consistente de reglas resulta ser más efectiva para que aprendan a reaccionar favorablemente a las normas establecidas por los adultos y para que su comportamiento resulte similar al de niños que reportan satisfacción por mostrar obediencia y conductas de autocontrol.

Específicamente, Cornell y Frick (2007) también encontraron un efecto de interacción entre las conductas inconsistentes de los padres y el temperamento de sus hijos que predijeron comportamientos característicos de baja empatía con la autoridad y bajos niveles de culpa por incumplimiento de reglas. Además, encontraron un efecto de interacción entre las conductas autoritarias de los padres y el temperamento de sus hijos como predictores de bajos niveles de culpa en el niño. Describieron que el castigo corporal, asociado con las conductas autoritarias de los padres, estuvo negativamente relacionado con la empatía. Los autores concluyeron que los padres de niños con problemas de conducta o alta desinhibición conductual, que utilizaron disciplina consistente, generaron niveles altos de reactividad en sus hijos y reportes de mayores sentimientos de culpa por incumplimiento de reglas así como mayor empatía con las figuras de autoridad. Finalmente, también confirmaron que ciertas prácticas de crianza basadas en el establecimiento de reglas y en estrategias de obediencia, que eviten el uso de castigo corporal, para niños con problemas de conducta desinhibida, fueron efectivas para reducir los problemas de conducta.

Sin embargo, Cornell y Frick (2007) reportaron que sus resultados no permiten afirmar que los niños con niveles extremos de desinhibición requieran estrategias diferentes de manejo conductual que las que necesitan los niños con alta reactividad a las normas y límites. Los autores asumen que la dificultad en alcanzar uno de sus objetivos tuvo relación con la carencia de medidas observacionales del comportamiento de los padres o de los niños, y a que solo utilizaron pruebas de autoreporte, que adicionalmente tuvieron bajos niveles de confiabilidad y validez.

Kochanska y Murray (2000) también han propuesto estrategias que promueven la socialización en niños categorizados como negativistas desafiantes que habían mostrado poca reactividad a la figura de autoridad o incluso carencia de emociones (miedo) ante la pérdida de reforzadores. Particularmente, observaron que el incremento en la interacción positiva entre padres e hijos basada en la cooperación, el apego y la mutua reciprocidad generaba mayor respuesta emocional ante las normas y límites y la ocurrencia de conducta pro-social en los niños.

Específicamente en México, Palacios y Andrade (2008) reportaron un estudio con adolescentes. Trabajaron con una muestra de mil participantes entre los 14 y los 22 años de edad para medir qué estilos parentales predecían comportamientos como conducta de consumo, antisocial y delictiva, entre otras. Los autores concluyeron que la supervisión materna y la imposición de normas por parte del 
papá se asociaron con la presencia o ausencia de conductas multi-problema en los adolescentes.

Sin embargo, existe poca literatura en México que describa, conductualmente, en qué consisten los estilos parentales de crianza que se relacionan con la ocurrencia del comportamiento negativista desafiante y agresivo en niños. Morales y Vázquez (2011) proponen la relevancia de evaluar el uso de procedimientos derivados de los principios básicos del comportamiento entre los que se encuentran el reforzamiento, el castigo, el control de estímulos y la extinción y su efecto sobre el comportamiento infantil.

Frick y Morris (2004), sin embargo, indicaron que el comportamiento derivado del temperamento difícil o desinhibido convierte a los niños en poco sensibles a las prohibiciones y sanciones de los padres y de otros agentes socializadores y que, por lo tanto, pueden fallar en internalizar reglas y normas. Cornell y Frick (2007) afirman que existen pocas teorías del desarrollo que integran el papel del temperamento o comportamiento desinhibido y el comportamiento de los padres para explicar el desarrollo de ciertos comportamientos y en particular el reporte de culpa o de la empatía en los niños con sus figuras de autoridad o su reactividad a los procedimientos de cambio conductual. Kochanska (1995) propone que el comportamiento infantil interactúa con el de los padres para provocar la reactividad del niño en su adherencia a las normas y límites.

En consecuencia se ha propuesto (Morales \& Vázquez, 2011) que las prácticas de crianza basadas en el afecto positivo y en la cooperación pueden resultar un componente crítico para el tratamiento de niños con problemas de conducta promoviendo el aprendizaje de la reactividad a procedimientos de manejo conductual (Cornell \& Frick, 2007). Es muy probable que los niños con comportamiento agresivo y negativista desafiante muestren cierto nivel de relación con ciertas prácticas de crianza y no con otras. Con el estudio de las prácticas de crianza, asociadas con determinados comportamientos, se podrían diseñar procedimientos de intervención más exitosos para los niños denominados como de difícil temperamento. El objetivo de este trabajo consiste en describir los estilos de crianza que se relacionan con el comportamiento negativista desafiante o agresivo en niños mexicanos por medio de la aplicación de autoreportes y sistemas de observación directa del comportamiento parental en situaciones simuladas por medio de un estudio descriptivo correlacional.

\section{Método}

\section{Participantes}

Se evaluó a 300 participantes de 18 entidades de la República mexicana, seleccionados por muestreo por cuota de instituciones de salud pública para la atención primaria a las adicciones, que asistieron voluntariamente a participar en el programa de crianza positiva por algún problema de conducta con alguno de sus hijos entre los 2 y los 12 años de edad, por lo que los grupos de la investigación no pudieron ser homogéneos en cantidad o en nivel académico. E1 $23 \%$ de los participantes provenían del estado de Veracruz, el $12 \%$, del Distrito Federal, el 9\%, de Jalisco, el $8 \%$, del Estado de México y para el resto de los estados la distribución porcentual se ubicó entre el $1 \%$ y $5 \%$ del total de la muestra para cada estado (Campeche, Chihuahua, Coahuila, Durango, Guanajuato, Guerrero, Hidalgo, Nayarit, Nuevo León, Puebla, San Luis Potosí, Tabasco, Tamaulipas y Tlaxcala).

El promedio de edad de los padres participantes fue de 34 años, con un rango entre los 18 y los 71 . El $92 \%$ de la población fue de sexo femenino. El $11 \%$ de la población era soltera, el $61 \%$, casada, el $20 \%$ vivía en unión libre, el $4 \%$, separada, el 3\%, divorciada y el $1 \%$, viuda. El $2 \%$ de los participantes no tenía estudios, el 16\% realizó la primaria, el $40 \%$, secundaria, el $24 \%$, preparatoria, el $16 \%$, era profesional y el $2 \%$ realizó un posgrado. El $63 \%$ se dedicaba al hogar, el $31 \%$ era empleado, el $5 \%$ era 
comerciante y el $1 \%$ era estudiante. El $46 \%$ de los niños de los padres participantes tenía entre 2 y 6 años y el 54\% entre 7 y 12 años. El $71 \%$ de los niños era de sexo masculino y el $29 \%$ de sexo femenino.

Los participantes firmaron un consentimiento informado donde se estableció la duración de la evaluación y que aceptaban que se utilizaran los resultados de su participación en el programa para investigación epidemiológica y difusión de resultados, cuidando plenamente su identidad y guardando la confidencialidad de la información, utilizando promedios grupales, pero que, no obstante, tenían derecho a declinar el uso de su información y participación en cualquier momento del estudio sin perjudicar su intervención en el plan de tratamiento posterior. El estudio no otorgó ningún tipo de incentivo a los participantes, pero se les explicó el beneficio social de su participación en la identificación de estrategias efectivas para la atención psicológica de diversas problemáticas sociales. Finalmente, se les otorgó información del contacto para recibir información requerida adicional.

\section{Instrumentos}

Se utilizaron dos grupos de instrumentos. Dentro de los psicométricos se encuentra el Inventario de Prácticas de Crianza, el Cuestionario de Habilidades de Manejo Infantil y el Inventario de Conducta Infantil. Finalmente, se utilizó un Sistema de Observación Directa de las conductas de interacción familiar.

El Inventario de Prácticas de Crianza (IPC; López, 2013) es un cuestionario autoaplicable de lápiz y papel, de 20 minutos de aplicación, que consta de 40 preguntas cerradas donde los padres tienen la posibilidad de elegir entre 7 opciones de respuesta, que van de nunca (0) hasta siempre (6); que evalúan las conductas de los padres respecto de la disciplina y a la promoción del afecto de sus hijos. El IPC fue validado con una muestra de 260 participantes y se obtuvo un nivel de confiabilidad de .92 (por medio del análisis de consistencia interna por alfa de Cronbach) y una varianza explicada del $64 \%$ (por medio del análisis factorial exploratorio) que arrojó 6 factores, por lo que el instrumento se evalúa por medio de 6 sub-escalas. La dimensión Castigo (con 9 reactivos y $52 \%$ de varianza explicada por ese único factor) se refiere a conductas no deseadas que los padres realizan para corregir el comportamiento de sus hijos; por ejemplo, gritar, regañar, obligar, etc. La dimensión Ganancias materiales (con 6 reactivos y $53 \%$ de varianza explicada por ese factor) se refiere a las consecuencias materiales que los padres otorgan ante el comportamiento deseado de sus hijos (objetos o juguetes). La dimensión Interacción social (11 reactivos y $64 \%$ de varianza explicada por ese factor) se refiere a las conductas que realizan los padres con la finalidad de tener interacciones positivas con sus hijos, como platicar, explicar, escuchar, etc. La dimensión de Normas (con 6 reactivos y $61 \%$ de varianza explicada por ese factor) evalúa acciones autoritarias que intentan los padres con el objetivo de establecer reglas a sus hijos; por ejemplo, imponer acuerdos o exigir el cumplimiento de las reglas. La dimensión Ganancias sociales (con 5 reactivos y $64 \%$ de varianza explicada por ese factor) se refiere a conductas de aprobación de los padres ante el comportamiento adecuado de sus hijos, como elogiar, felicitar, dar las gracias, etc. Finalmente la dimensión Límites (con 3 reactivos y $63 \%$ de varianza explicada por ese único factor) se refiere a la imposición que los padres realizan para establecer límites a sus hijos; por ejemplo, establecer autoridad o imponer un horario.

El Cuestionario de Habilidades de Manejo Infantil, basado en situaciones hipotéticas de crianza (CHAMI; Morales \& Vázquez, 2011), es un cuestionario autoaplicable de lápiz y papel con 10 viñetas o situaciones simuladas de evaluación sobre las habilidades de manejo de conducta infantil. Son situaciones hipotéticas de interacción problemática con el niño, en el que los padres responden, de manera abierta, qué harían ante dicha situación. Cada viñeta es calificada sobre la base 
de tres posibilidades: 0 si el padre no describe la habilidad, 1 si la describe parcialmente, o 2 si la describe completamente. El instrumento fue validado con 294 participantes de distintos estados del país, se obtuvo una consistencia interna por alfa de Cronbach de 62 y una varianza explicada del $55 \%$, por medio del análisis factorial exploratorio que arrojó 4 factores. También se obtuvo la fiabilidad promedio entre evaluadores entrenados en el código de calificación, siempre mayor al 80\%. La concordancia se obtuvo a partir del cálculo de los Acuerdos/Acuerdos + desacuerdos X 100. Entonces, el instrumento quedó constituido por 4 sub-escalas. La dimensión Ignorar como técnica para promover conducta adecuada (ITCA; con 3 reactivos y $57 \%$ de varianza explicada por ese único factor); la dimensión Elogio (con 2 reactivos y $62 \%$ de varianza explicada por ese factor); la dimensión Instrucciones claras, solución de problemas y establecimiento de reglas (ICSE; con 3 reactivos y $55 \%$ de varianza explicada por ese factor) y la dimensión de interacción social-académica (ISA; con 2 reactivos y $67 \%$ de varianza explicada por ese factor).

El Inventario de Conducta Infantil (ICI; Morales \& Martínez, 2013) es un instrumento autoaplicable de lápiz y papel de 12 reactivos. La consistencia interna del instrumento fue de .86 (por alfa de Cronbach) y la varianza explicada del $60 \%$ (por medio del análisis factorial exploratorio), que arrojó dos factores que pueden resolverse aproximadamente en 15 minutos. En la dimensión de comportamiento oposicionista desafiante, el padre señala el grado o intensidad con el que se presentan comportamientos infantiles característicos del desorden negativista desafiante con base en el DSM-V (APA, 2013) en 8 reactivos $(60 \%$ de varianza explicada para ese único factor) con una escala Likert de 5 puntos que va de Nunca (0) a Siempre (4). Los reactivos evalúan la presencia de berrinches, discusiones, oposición, molestar a otros, culpar, irritación, enojo y comportamiento vengativo. En la dimensión de comportamiento agresivo, el padre señala el grado o intensidad con el que se presenta dicho comportamiento verbal y físico (Morales, 2002) en 4 reactivos (58\% de varianza explicada para ese factor) con una escala Likert de 5 puntos.

El Sistema de Observación Directa (Morales, 2001; Morales \& Martínez, 2013) estuvo constituido por tres listas cotejables de ocurrencia de evento, dos registros de evento y tres registros de intervalo parcial de tiempo. Las tres listas cotejables evaluaron la corrección simple del comportamiento, el establecimiento de reglas y la solución de problemas. Las listas de corrección simple se puntuaron del 0 (no realiza la habilidad) o 1 (reprende por medio de corrección simple, instrucciones claras, extinción del comportamiento no deseado, instigamiento, solución de problemas, establecimiento de contingencias, costo de respuesta y reforzamiento positivo) acorde a la situación. La lista cotejable de establecimiento de reglas evalúa nueve conductas: menciona las reglas, pide al niño que las repita, establece ganancia, establece pérdida, elogia conducta deseada, reprimenda, corrección, ignora protestas y utiliza pérdida de privilegios. Se obtiene el porcentaje de ocurrencia de las conductas. La lista cotejable de solución de problemas evaluó siete conductas: descripción del problema, explica los porqué de un problema, pregunta, sugiere opciones, evalúa, elige y consolida. Se obtuvo el porcentaje de respuestas correctas por regla de tres.

Los dos registros de evento evaluaron el elogio de conducta académica y el seguimiento instruccional. En el último se evalúan las conductas de interacción del padre: obtiene atención, da instrucción clara, espera, elogia, vuelve a obtener atención, repite instrucción clara, cambia tono de voz, espera por segunda ocasión e instiga. En el registro de elogio se obtiene el porcentaje de dicha conducta y en seguimiento instruccional, el porcentaje por oportunidad de ocurrencia.

Los tres registros de intervalo parcial de $10 \mathrm{se}$ gundos durante 5 minutos evaluaron interacción social, interacción académica y enseñanza inci- 
dental (Morales \& Martínez, 2013). El registro de interacción social evaluó las siguientes conductas: compartir, elogiar, risa provocada, mirar, sonreír, reír, tocar y peticiones verbales. El registro de interacción académica y el de enseñanza incidental evaluó, además de las anteriores, instrucción clara, modelar, instigar y corregir. En los tres se obtiene el porcentaje parcial de intervalos por conducta.

En todos los registros se obtuvo el porcentaje de acuerdo con dos observadores independientes entrenados en observación directa en los registros y solo se consideraron confiables aquellos registros cuya concordancia fue mayor al $80 \%$. La concordancia también se obtuvo a partir del cálculo de los Acuerdos/Acuerdos + desacuerdos X 100. A partir de las listas cotejables, de los registros de evento y de los registros de intervalo parcial se obtuvieron los porcentajes de la conducta meta o de los intervalos registrados por conducta y promedios globales de interacción. Para la definición operacional de las conductas se puede revisar el material suplementario de este artículo.

\section{Procedimiento}

Se utilizó un estudio no experimental de tipo correlacional por medio de dos sesiones de evaluación. En cada una de las entidades participantes se había convocado a los participantes a ingresar en un programa de entrenamiento conductual a padres, por lo que, previo a ello, se les convocó a las dos sesiones de evaluación de 120 minutos cada una, que constituyen el actual estudio. En la primera sesión, los participantes recibieron los cuestionarios psicométricos descritos en el apartado de instrumentos y de forma grupal se dieron las siguientes instrucciones:

En esta sesión se realizarán una serie de cuestionarios que nos permitirán conocer las habilidades con las que ustedes cuentan para corregir a sus hijos en este momento y la frecuencia con la que se observan ciertas conductas en ellos. El llenado de los cuestionarios es individual y comenzaremos por completar el inventario de prácticas de crianza que tiene como objetivo conocer lo que ustedes hacen con relación al comportamiento de sus hijos e hijas. Por lo que a continuación encontrarán una serie de palabras que describen algunas conductas que los padres y madres realizan cuando quieren llevarse bien con sus hijos, sus hijos no hacen lo que se les dice, sus hijos obedecen y cuando quieren establecerles reglas. Por lo que deberán marcar con una $\mathrm{X}$ sobre el cuadro que representa mejor la frecuencia con la que llevan a cabo esa conducta, tomando en consideración que cuanto más grande y más cerca de la palabra se encuentre, el cuadro indica que siempre realiza esa conducta. En el ejemplo podrán ver que hay 6 cuadros y una línea después de la frase "ir al cine". Si marcan el cuadro más grande, esto indica que ustedes siempre van al cine. Si marcan la línea, significa que ustedes nunca van al cine. El resto de los cuadros indican diferentes grados de frecuencia, utilicen estos para encontrar la frecuencia con la que llevan a cabo determinada conducta con sus hijos. El segundo Cuestionario de Habilidades de Manejo Infantil, basado en situaciones hipotéticas de crianza, es un cuestionario de 10 preguntas abiertas en el que solo les pediremos que escriban con el mayor detalle posible lo que normalmente harían si su hijo mostrara dicha situación. Es importante que piensen cómo actuarían ante esa situación. Si tienen alguna duda, tengan la plena confianza de preguntar y aclararla. El tercer instrumento es un inventario que se refiere a la conducta de sus hijos, en él ustedes van a elegir la opción de respuesta que mejor describa la intensidad con la que se presenta la conducta de su hijo actualmente. ¿Tienen alguna pregunta? Podemos comenzar.

En la segunda sesión de 120 minutos se llevó a cabo una evaluación de situaciones simuladas por medio del sistema de observación directa, con ocho grupos de estímulos relacionados con las habilidades y conductas de interacción de los padres, todas ellas a cargo de un evaluador que presentó 
los disparadores específicos por medio de un ensayo conductual.

El primer grupo de estímulos que presentó el evaluador a los padres estuvo constituido por 10 situaciones de evaluación sobre corrección del comportamiento infantil ante la entrega de reportes escolares, la hora de la comida, en el supermercado, la pelea entre hermanos y la entrega del elogio a conducta deseada con duración máxima de 15 segundos por cada estímulo. El segundo grupo de estímulos estuvo constituido por la presentación de obediencia a diez instrucciones académicas para la evaluación del reforzamiento positivo (elogio) ante la obediencia en esta situación. El tercer grupo de estímulos estuvo constituido por la oportunidad de ocurrencia de conductas de interacción social y la entrega contingente de la imitación por el evaluador en su papel de niño del comportamiento realizado por el padre. El cuarto grupo de estímulos estuvo constituido por la oportunidad de obediencia a 10 instrucciones que se solicitaba al padre dar. El evaluador en su papel de niño mostraba obediencia si el padre seguía el formato de instrucciones claras o desobedecía si el padre omitía alguno de los pasos de la instrucción clara. El quinto grupo de estímulos estuvo constituido por el establecimiento de reglas en una situación simulada a la hora de la comida. El sexto grupo de estímulos estuvo constituido por la evaluación de habilidades a la hora de la tarea, donde el evaluador realizaba correctamente tres sumas y se equivocaba en otras dos durante el ensayo conductual. El séptimo grupo de estímulos estuvo constituido por la evaluación de la situación de enseñanza incidental durante un momento cotidiano en la vida familiar: la preparación del agua de limón. El octavo grupo de estímulos estuvo constituido por la evaluación de la situación de solución de problemas para mantener la habitación del niño arreglada.

Para la realización de los ensayos conductuales de las situaciones simuladas se otorgaron las siguientes instrucciones:
A continuación realizaremos una serie de situaciones de evaluación en las que yo jugaré el papel de un niño. Sé que es un poco inusual para usted este tipo de evaluación, pero lo importante de estos ensayos consiste en brindarle la oportunidad de mostrar las herramientas con que cuenta actualmente para resolver la conducta de su hijo. Por eso le pido que procure responder de la forma más natural posible, y sobre todo que muestre cómo reaccionaría, en estos momentos, ante la situación determinada. Esta actuación es confidencial, y los resultados, al igual que aquellos obtenidos en los cuestionarios, también son confidenciales. Usted puede tener la confianza de mostrar, con su comportamiento, lo que haría, en este momento, si su hijo se comportará como yo voy a hacerlo. Aunque sea un poco extraño para usted, por favor, es importante que procure imaginar que yo soy un niño (su hijo, si es posible) y actúe y diga lo que considere necesario para resolver las situaciones que se le presenten. ¿Tiene alguna duda? Comencemos.

\section{Análisis estadísticos}

Para el análisis de los datos, se llevaron a cabo los análisis descriptivos de cada variable predictora (estilos de crianza) sobre las pronosticadas (oposición y agresión) medidas psicométricamente y por medio de la observación directa. Es decir, se obtuvieron las medias como medida de tendencia central y las desviaciones estándar como medidas de dispersión. Para estimar el efecto de una variable sobre otra se utilizó el modelo de regresión lineal múltiple, relacionado con el coeficiente $r$ de Pearson que indica la varianza de factores comunes. Todos los análisis se realizaron por medio del paquete estadístico SPSS ${ }^{\circledR}$ versión 15.0 para Windows ${ }^{\circledR}$. Se estableció un $95 \%$ de confianza para el valor de certeza de los datos. Es decir, estableció a priori que los niveles de significación debían ser menores al .05 , para dar sentido a los resultados. 


\section{Resultados}

Primero se muestra el porcentaje de participantes que presentaron algún posible diagnóstico de comportamiento negativista desafiante o agresión infantil, luego los porcentajes promedio de conducta negativista desafiante y agresión, prácticas de crianza, habilidades de manejo conductual y, finalmente, el efecto predictivo de las prácticas de crianza, a partir de las regresiones lineales, sobre el puntaje de comportamiento infantil, desde estas habilidades reportadas y observadas en los padres de familia.

En cuanto al porcentaje de participantes que superaron el porcentaje de comportamientos mínimo necesario para alcanzar el criterio diagnóstico a partir del DSM-V (APA, 2013), se encontró que el $39.4 \%$ de los participantes reportó que sus hijos presentaron al menos cuatro comportamientos o más del orden negativista desafiante, siempre en los últimos seis meses ( $51 \%$ de los comportamientos posibles con puntuaciones 5 de la escala Likert) y que el $17.2 \%$ de los participantes reportó que sus hijos presentan más del $51 \%$ de comportamiento agresivo siempre.

En la tabla 1 se pueden observarlos promedios y desviaciones estándar de los porcentajes por escalas y de los sistemas de observación directa. Como se puede ver, el promedio de conducta problema (ICI) más alto fue para el comportamiento oposicionista desafiante $(M=43.86)$, seguido por la agresión infantil $(M=29.09)$. También se observan los promedios de las escalas del Inventario de Prácticas de Crianza (IPC), donde el porcentaje promedio más alto fue para interacción social $(M=80.77)$, seguida por el de Normas $(M=79.24)$, Ganancias sociales $(M=78.04)$, el Castigo $(M=75.21)$ y Límites $(M=73.21)$. Se observan también los porcentajes promedio del Cuestionario de Manejo Infantil (CHAMI), donde la escala con el promedio más alto fue Elogio $(M=39.18)$, seguida por Interacción social académica $(M=36.49)$, instrucciones claras, solución de problemas y estableci- miento de reglas $(M=28.47)$ e ignora como técnica para promover conducta adecuada $(M=23.88)$. También se muestran los porcentajes promedio de los comportamientos observados por medio del sistema de registros (OBS). El porcentaje promedio más alto fue para Seguimiento instruccional $(M=43.59)$, seguido por Solución de problemas $(M=38.52)$, Establecimiento de reglas $(M=36.56)$, Elogio para conducta académica $(M=28.61)$, Interacción social $(M=14.92)$, Interacción académica $(M=12.89)$, Corrección simple $(M=16.67)$ y Enseñanza incidental $(M=7.98)$.

Se llevaron a cabo análisis de varianza para cada una de estas variables predictoras y pronosticadas con la finalidad de evaluar el efecto por género del niño y por nivel educativo de los padres. No se encontraron diferencias significativas por sexo en ninguna de las variables de estudio. Hubo diferencias para una escala del IPC: interacción social; y dos escalas del CHAMI: ITCA e ICSE por nivel educativo de los padres. Particularmente en la escala de interacción social, los padres con nivel educativo de primaria tuvieron un promedio menor $(M=13.05)$ de habilidades que específicamente los padres con licenciatura $(M=23.86 ; F(5,298)$ $=2.67, p=.036$ ) de acuerdo con los estudios $p o s$ hoc. En la escala ITCA, los padres con nivel educativo de primaria tuvieron un promedio menor $(M=17.36)$ para usar la técnica de ignorar, que específicamente los padres con licenciatura $[M=$ $32.98 ; F(5,298)=2.36, p=.04]$. En la escala ICSE los padres con nivel educativo de primaria tuvieron un promedio menor $(M=19.1)$ de habilidades para usar las instrucciones claras, establecimiento de reglas y solución de problemas, que específicamente los padres con licenciatura $(M=36.88 ; F(5,298)$ $=2.35, p=.04)$. No hubo diferencias significativas por género o escolaridad para el resto de las nueve escalas psicométricas ni en las ocho del sistema de observación directa.

En la tabla 2 se pueden ver los resultados de los análisis de regresión sobre el comportamiento negativista desafiante. Las variables asociadas signi- 
Tabla 1

Promedio, desviación estándar, rango, sesgo y curtosis en las escalas psicométricas y sistema de observación directa

\begin{tabular}{|c|c|c|c|c|c|}
\hline Escala o sistema de observación & $M(D T)$ & Rango & & & \\
\hline & & Mínimo & Máximo & Sesgo & Curtosis \\
\hline \multicolumn{6}{|l|}{ ICI } \\
\hline Comportamiento oposicionista desafiante & $43.86(21.52)$ & 6.25 & 87.5 & .10 & -.94 \\
\hline Agresión infantil & $29.09(23.51)$ & 28.13 & 75 & .54 & -.52 \\
\hline \multicolumn{6}{|l|}{ IPC } \\
\hline Castigo & $75.21(16.76)$ & 11.11 & 100 & -.84 & .24 \\
\hline Ganancias Materiales & $58.71(21.71)$ & 5.55 & 100 & -.21 & -.78 \\
\hline Interacción social & $80.77(17.12)$ & 22.73 & 100 & -1.34 & 1.39 \\
\hline Normas & $79.24(19.17$ & 16.67 & 100 & .14 & .28 \\
\hline Ganancias Sociales & $78.04(20.25)$ & 6.67 & 100 & -1.15 & 1.02 \\
\hline Límites & $73.21(22.08)$ & 0 & 100 & -.78 & -.21 \\
\hline \multicolumn{6}{|l|}{ СНАМI } \\
\hline ITCA & $23.88(26.47)$ & 0 & 100 & .93 & -.04 \\
\hline Elogio & $39.18(23.62)$ & 0 & 100 & .44 & .13 \\
\hline ICSE & $28.47(27.07)$ & 0 & 100 & .64 & -.71 \\
\hline ISA & $36.49(27.14)$ & 0 & 100 & .32 & -.64 \\
\hline \multicolumn{6}{|l|}{ OBS } \\
\hline Corrección simple & $16.67(14.78)$ & 0 & 80 & 1.18 & 1.29 \\
\hline Elogio & $28.61(20.47)$ & 0 & 80 & .46 & -.68 \\
\hline Interacción social & $14.92(11.77)$ & 0 & 47.47 & .65 & .09 \\
\hline Seguimiento Instruccional & $43.59(26.42)$ & 1.11 & 92.20 & .23 & -1.35 \\
\hline Establecimiento de Reglas & $36.56(26.13)$ & 0 & 89 & .21 & -1.13 \\
\hline Interacción académica & $12.89(10.44)$ & 0 & 58 & 1.51 & 3.6 \\
\hline Enseñanza incidental & $7.98(8.83)$ & 37 & 48 & 2.25 & 5.71 \\
\hline Solución de problemas & $38.52(23.95)$ & 0 & 86 & .15 & -.74 \\
\hline
\end{tabular}

Nota: IPC-Inventario de Prácticas de crianza; CHAMI-Cuestionario de Habilidades de Manejo Infantil; ICI- Inventario de Conducta Infantil; OBS- Sistemas de Observación Directa.

ficativamente con el comportamiento oposicionista fueron el porcentaje promedio de Castigo $(R 2=.21$; $F(1,298)=78.09, p<.000)$, la escala de interacción social $(\mathrm{IPC} ; R 2=04(F(1,298)=12.88, p<.000)$, las viñetas de ignorar como Técnica para promover comportamiento adecuado (ITCA; $R 2=.05(F(1$, $298)=16.32, p<.000)$, las de instrucciones claras, solución de problemas y establecimiento de reglas $(\mathrm{ICSE} ; R 2=.01 F(1,298)=4.26, p=.04)$, las de interacción social académica (ISA; $R 2=.02 F(1$, $298)=6.14, p=.01), \mathrm{y}$ los porcentajes promedio de conductas del padre de interacción social (elogiar, mirar, reír, y tocar; $R 2=.09(F(1,298)=2.74$, $p=.03$ ), seguimiento instruccional (esperar, elo- 
giar y volver a obtener la atención; $R 2=.10(F(1$, $298)=3.30, p=.013)$, establecimiento de reglas $(R 2=.04(F(1,298)=4.84, p=.03)$ y solución de problemas $(R 2=.06(F(1,298)=7.38, p=.008)$. El modelo de regresión múltiple sobre el comportamiento oposicionista desafiante arrojó una $R 2=.42$ $(F(1,298)=5.06, p<.000)$.

Tabla 2

Predictores significativos del comportamiento negativista desafiante

\begin{tabular}{lrcrr}
\hline \multicolumn{1}{c}{$\begin{array}{c}\text { Escala o sistema de } \\
\text { observación }\end{array}$} & $R 2$ & $B$ & $F(1,298)$ & $p$ \\
\hline Castigo & 0.21 & 0.457 & 78.09 & .000 \\
Interacción social & 0.04 & -0.205 & 12.88 & .000 \\
ITCA & 0.05 & -0.229 & 16.32 & .000 \\
ICSE & 0.01 & -0.119 & 4.26 & .04 \\
ISA & 0.02 & -0.143 & 6.14 & .01 \\
Interacción social & 0.09 & -0.147 & 2.74 & .03 \\
Seguimiento instruccional & 0.1 & -0.293 & 3.3 & .013 \\
Establecimiento de reglas & 0.04 & -0.198 & 4.84 & .03 \\
Solución de problemas & 0.06 & -0.242 & 7.38 & .008 \\
\hline
\end{tabular}

Finalmente, en la tabla 3 se puede observar que el grupo de variables asociadas al comportamiento de agresión estuvo constituido por el porcentaje promedio de Castigo $(R 2=.12 ;(F(1,298)=38.96$, $p<.000$ ), la escala de interacción social (IPC; $R 2=$ $.04(F(1,298)=10.63, p=.001)$, las viñetas de ignorar como Técnica para promover comportamiento adecuado $($ ITCA; $R 2=.04 ; F(1,298)=11.13$, $p=.001)$, las de instrucciones claras, solución de problemas y establecimiento de reglas (ICSE; $R 2=$ $.02(F(1,298)=4.87, p=.028)$ y las de interacción social académica $($ ISA; $R 2=.02(F(1,298)=6.73$, $p=.010)$ y de los porcentajes promedio de conductas del padre de interacción social (elogiar; $R 2=$ $.06(F(1,298)=7.47, p=.007)$ y de seguimiento instruccional (esperar; $R 2=.04(F(1,298)=4.94$, $p=.028)$. El análisis de regresión lineal múltiple mostró una $R 2=.29(F(1,298)=6.70, p<.000)$.
Tabla 3

Predictores significativos del comportamiento de agresión

\begin{tabular}{lcccc}
\hline \multicolumn{1}{c}{$\begin{array}{c}\text { Escala o sistema de } \\
\text { observación }\end{array}$} & $R 2$ & $B$ & $F(1,298)$ & $p$ \\
\hline Castigo & 0.12 & 0.342 & 38.96 & .000 \\
Interacción social & 0.04 & -0.186 & 10.63 & .001 \\
ITCA & 0.04 & -0.191 & 11.13 & .001 \\
ICSE & 0.02 & -0.127 & 4.87 & .028 \\
ISA & 0.02 & -0.149 & 6.73 & .01 \\
Interacción social & 0.06 & -0.243 & 7.47 & .007 \\
Seguimiento instruccional & 0.04 & -0.179 & 4.94 & .028 \\
\hline
\end{tabular}

\section{Discusión}

El objetivo del trabajo consistió en describir los estilos de crianza que se relacionan con el comportamiento negativista desafiante y agresivo en niños mediante de la aplicación de autoreportes y la metodología observacional del comportamiento de los padres en situaciones simuladas de interacción, por medio de un estudio descriptivo correlacional. En primer lugar, los resultados indicaron que el porcentaje de niños con comportamiento negativista desafiante fue mayor que el de niños reportados con conducta agresiva (Medina Mora et al., 2003). Sin embargo, se tuvo la oportunidad de trabajar con una muestra de niños cuyos padres reportaron niveles variables de conducta desinhibida tal como el berrinche, la discusión con adultos, la oposición, la desobediencia, la irritabilidad, el enojo, el resentimiento hacia las figuras de autoridad y específicamente de agresión física y verbal.

Las prácticas de crianza reportadas con mayores porcentajes fueron aquellas denominadas como de baja efectividad (Scahill et al., 2006). Esto es, los padres reportaron altos promedios del uso del castigo, la entrega de ganancias materiales y el establecimiento de normas y límites poco claros. Los padres reportaron promedios bajos de prácticas de crianza basadas en técnicas efectivas tales 
como el ignorar conducta no deseada y corrección simple del comportamiento, el elogio de la obediencia y de la actividad académica, y ejecutaron bajos porcentajes promedio de interacción social, instrucciones claras, interacción social académica, establecimiento de reglas, enseñanza incidental y solución de problemas (Hawes \& Dadds, 2005; Morales \& Vázquez, 2011). Sin embargo, también se reportaron algunas prácticas de crianza positiva de alto porcentaje promedio como la interacción social (Morales \& Vázquez, 2011) y la entrega de ganancias sociales.

El factor de riesgo que se asoció confiablemente con el reporte de comportamiento negativista desafiante y el agresivo fue el uso de castigo. Con ello, el presente hallazgo parece apoyar la premisa de que el uso de técnicas de castigo se asocia con la presencia de conducta antisocial y negativista en la infancia (Cornell \& Frick, 2007). Podría suceder que la conducta antisocial, como los berrinches, la discusión con adultos, la oposición, la desobediencia, la irritabilidad, el enojo, el resentimiento hacia las figuras de autoridad y la conducta agresiva física o verbal estén asociadas con un mayor reporte de uso de estrategias inefectivas de corrección por parte de los padres (Barkley, 1998; Cornell \& Frick, 2007; Frick, 2008) como lo es el castigo físico. Wootton, Frick, Shelton \& Silverthorn (1997) concluyeron que los niños con altos niveles de problemas de conducta tienden a mostrar insensibilidad o falta de reacción, y por lo tanto menor emoción o menos estrés por los efectos de su conducta en sí mismos o sobre otros a partir de las correcciones aplicadas por los padres (Frick \& White, 2008). El presente estudio mostró que los bajos índices de conductas de interacción social, o en situación académica, de seguimiento instruccional para fomentar la obediencia, de establecimiento de reglas y de solución de problemas, aunados a la baja frecuencia del uso de la técnica de ignorar conducta no deseada, también se asociaron confiablemente con el comportamiento negativista desafiante, confirmando lo que Wootton et al. (1997) reportaron. Esto es, una fuerte asociación entre el bajo nivel de prácticas de crianza efectivas y el reporte de los problemas de conducta con niños que podrían presentar altos niveles de insensibilidad, y cuyos padres reportaron la presencia de un fuerte reto a la autoridad.

Hawes y Dadds (2005) señalaron que serán más exitosos los programas de tratamiento basados en las estrategias conductuales de manejo de obediencia, interacción social positiva y técnicas de corrección del comportamiento particularmente en aquellos niños con el trastorno negativista desafiante. En este sentido, los resultados del actual estudio mostraron esta relación inversa entre la presencia de las prácticas de crianza, basadas en los procedimientos conductuales, con el reporte de conducta desinhibida o negativista desafiante o agresiva. Particularmente, se observó el uso de conductas positivas en la interacción social y el uso de conductas de seguimiento instruccional para la promoción de la obediencia infantil que se asociaron con un reporte de menor grado de conducta negativista desafiante y agresiva, permitiendo una posible alusión al planteamiento de Hawes y Dadds (2005) de que específicamente los niños con altos niveles de insensibilidad emocional pueden mostrar reactividad a las estrategias disciplinarias como las encontradas en el presente estudio.

El reporte de las prácticas de crianza basadas en el reforzamiento positivo, control de estímulos, corrección simple del comportamiento y en la técnica de ignorar conducta no deseada se asociaron también con un menor porcentaje de reportes de conducta agresiva como negativista y desafiante. Este resultado puede ser consistente con el estilo de respuesta orientada a la recompensa, que parece ser característica de niños con alta insensibilidad emocional (Cornell \& Frick, 2007; Waschbusch, Carrey, Willoughby, King \& Andrade, en prensa). En este sentido, Ayala et al. (2002) demostraron que las familias con posibilidad de realizar actividades recreativas y la orientación a metas de vida predicen un menor porcentaje de conducta agresiva. En el presente estudio, se evaluaron conductas de 
los padres que podrían insertarse conductualmente en la categoría de acciones orientadas a mejorar la vida y alcanzar las metas familiares. Por lo que parece, los resultados pueden ser congruentes con dicha afirmación.

Frick y Morris (2004) sugirieron que algunos niños que muestran comportamiento derivado del temperamento difícil, tal como el desorden negativista desafiante o el comportamiento agresivo, son poco sensibles a las prohibiciones y sanciones de los padres y de otros agentes socializadores y por lo tanto pueden fallar en internalizar reglas y normas. Sin embargo, los resultados del presente estudio parecen sugerir que el comportamiento de los padres basados en los procedimientos de cambio conductual podrían estar asociados con la reactividad de los niños con problemas de conducta ante el establecimiento de reglas y normas claras así como a mostrar comportamiento empático ante las figuras de autoridad como lo sugieren Cornell y Frick (2007). Kochanska (1995) propone que el comportamiento infantil interactúa con el de los padres para provocar la reactividad del niño en su adherencia a las normas y límites. Los resultados del presente estudio indicaron que las conductas justamente de interacción social positiva, como las de seguimiento instruccional, establecimiento de reglas y solución de problemas predijeron estadísticamente un menor comportamiento de berrinche, enojo u oposición. Aparentemente, los niños sin problemas de conducta podrían estar reaccionando más fácilmente al establecimiento de normas. Para los niños con problemas de conducta, la disciplina flexible y basada en la conducta positiva de los padres podría estar promoviendo un nivel óptimo de reacción o malestar que favorecería la autoeficacia y el procesamiento efectivo de los mensajes de la socialización de los padres. Sin embargo, solo investigación adicional con análisis predictivos y modelos que permitan controlar el efecto de las variables independientes, a partir de una mayor fortaleza estadística, permitirá confirmar dicha premisa.
En este estudio, los padres también reportaron que sus hijos con problemas de conducta parecen no experimentar un nivel óptimo de reacción en situaciones que involucran el castigo potencial, ya que la relación fue directamente proporcional, es decir a mayor uso del castigo, mayor reporte de conducta negativista desafiante y agresiva. Es posible que el reporte del uso de castigo y su nivel de asociación con el reporte de conducta antisocial en los niños indique la carencia de la internalización o reacción a las normas establecidas (Cornell \& Frick, 2007). Una investigación futura deberá abordar la medición de las conductas características de los niños que disfrutan de romper las reglas o que no muestran emoción asociada al cumplimiento de normas. No obstante, los resultados del presente estudio permiten considerar la posibilidad de que los niños con problemas de conducta requieran que sus padres utilicen un grupo de reglas más consistentes, al observar una relación inversa entre el uso de esta estrategia y un menor porcentaje de comportamiento negativista y oposicionista. En consecuencia, se apoya la propuesta de que las prácticas de crianza basadas en el afecto positivo y en la cooperación puede resultar ser un componente crítico para el tratamiento de niños con problemas de conducta (Cornell \& Frick, 2007; Morales \& Vázquez, 2011) pero investigación adicional deberá abordar el estudio del nivel de aprendizaje de la reactividad a procedimientos de manejo conductual.

En este estudio, los padres de niños con problemas de conducta reportaron y ejecutaron estrategias de crianza que podrían estar asociadas a una posible mayor reactividad de sus hijos a estas prácticas de crianza, particularmente, a la interacción social positiva, al uso de la técnica de ignorar, al uso de las instrucciones claras, la solución de problemas, el establecimiento de reglas y la interacción académica positiva (Cornell \& Frick, 2007). Los resultados dirigen la atención al estudio de la reactividad de los niños a estas prácticas de crianza y no a otras tales como la ejecución de la corrección simple o la sola entrega de ganancias materiales. 
En el presente estudio, las conductas de los padres se asociaron con mayor frecuencia a las medidas de reactividad (menor oposición) y empatía (menor resistencia a la autoridad) en los niños señalados por mostrar problemas de conducta $y$ desinhibición. Los efectos benéficos de experimentar conductas consistentes en los padres enfatiza una aproximación orientada por el establecimiento de reglas y por la demanda de obediencia para niños con problemas de conducta por desinhibición (Hawes \& Dadds, 2005). Sin embargo, queda abierta la posibilidad de estudiar aquellos elementos que permitan saber si los niños con niveles extremos de desinhibición requieren estrategias similares de manejo conductual que los niños con alta reactividad a las normas y límites. Una investigación adicional permitirá confirmar este argumento.

No obstante, es importante señalar que los hallazgos pueden considerarse novedosos al haber hecho uso de medidas observacionales del comportamiento de los padres y al uso de pruebas de autoreportes confiables y válidos. Los hallazgos constituyen, entonces, un primer acercamiento para conocer el proceso involucrado en el desarrollo de conductas características de la reactividad a las reglas y límites claros. Una investigación adicional permitirá describir cómo el comportamiento de los padres y su óptimo ajuste al comportamiento de los niños dependen del nivel de reactividad ante dicha interacción.

En conclusión, los resultados parecen indicar que particularmente los niños que presentan problemas de conducta hasta el trastorno negativista desafiante o comportamiento agresivo, podrían no mostrar un nivel óptimo de reacción en situaciones que involucran el castigo potencial y por ende podrían no internalizar las normas o reaccionar exitosamente a las reglas por este medio de crianza. También parece que los niños que muestran comportamiento desinhibido podrían estar requiriendo que sus padres utilicen prácticas más consistentes y firmes que promuevan una reacción más efectiva, pues se observa una reducción en el reporte de los síntomas asociados con las conductas de interacción social en los padres, de establecimiento de reglas, de instrucciones claras y de solución de problemas. Y sin embargo, también es muy probable que ciertas prácticas como el castigo promuevan un efecto contraproducente en estos niños y por ende una reducida autoeficacia. Investigación adicional deberá abordar esta última premisa.

La contribución a la psicología traslacional consiste en conocer los principios básicos que pueden ser representados en los procedimientos conductuales o "buenas prácticas" de intervención en escenarios clínicos reales para conductas particulares (Morales, 2012; Santoyo, 2012). La identificación de las prácticas de crianza asociadas al comportamiento agresivo o negativista desafiante permitirán diseñar procedimientos de intervención menos costosos y más efectivos para los niños denominados como de temperamento difícil. Con ello se fomentará, probablemente, que los niños con comportamiento desafiante muestren mayor reactividad a las figuras de autoridad y mayor comportamiento representativo de la interiorización de normas y límites claros del comportamiento, reduciendo la progresión del comportamiento hacia actos delictivos y de la violación de propiedad privada (Frick \& White, 2008) como los que hoy en día se dispersan preocupantemente en nuestra población. Las intervenciones deben enfocarse en el proceso temprano del desarrollo que precede a los problemas de conducta, previniendo el surgimiento y mantenimiento de conducta agresiva y antisocial en etapas posteriores de la vida.

\section{Referencias}

American Psychiatric Association (2005). Diagnostic and Statistical Manual of Mental Disorders $\left(4^{\text {th }}\right.$ Edition). Washington DC: American Psychiatric Publishing. 
American Psychiatric Association (2013). Diagnostic and Statistical Manual of Mental Disorders ( $5^{\text {th }}$ Edition). Arlington, VA: American Psychiatric Publishing.

Ayala, V. H. E., Pedroza, C. F., Morales, C. S., Chaparro, C. L. A., \& Barragán, T. N. (2002). Factores de riesgo, factores protectores y generalización del comportamiento agresivo en una muestra de niños en edad escolar. Salud Mental, 25(3), 27-40.

Baker B. L., \& Feinfeld. K. A. (2007). Early intervention and parent education. In: A. Carr, G. O'Reilly, P. N. Walsh \& J. McEvoy (Eds.), Handbook of Intellectual Disability and Clinical Psychology Practice (pp. 336-370). New York, NY: Brunner-Routledge, Taylor and Francis Group.

Barkley, R. A. (1997). Defiant Children. A Clinician's Manual for Assessment and Parent Training. New York: The Guilford Press.

Bradley, S., Jadda, D., Brody, J., Landy, S., Tallet, S., Watson, W., \& Stephens, D. (2003). Brief psycho educational parenting program: An evaluation and 1 year follow-up. Journal of American Academic of Child and Adolescent Psychiatry, 42, 1171-1178.

Clark, D. B., Vanyukov, M., \& Cornelius, J. (2002). Childhood Antisocial Behavior and Adolescent Alcohol Use Disorders. Recuperado de http:// pubs.niaaa.nih.gov/publications/arh26-2/109115.htm.

Cornell, A. H., \& Frick, P. J. (2007). The moderating effects of parenting styles in the association between behavioral inhibition and parent-reported guilt and empathy in preschool children. Journal of Clinical Child and Adolescent Psychology, 36, 305-318.doi: 10.1080/15374410701444181

Dadds, M. R., Maujean, A., \& Fraser, J. A. (2003). Parenting and conduct problem in children: Australian data and psychometric properties of the Alabama parenting questionnaire. Australian Psychologist, 38, 238-241. doi: 10.1080/00050060310001707267
Fajardo, V. V., \& Hernández, G. L. (2008). Tratamiento cognitivo conductual de la conducta agresiva infantil. Revista Mexicana de Análisis de la Conducta, 34(2), 371-389.

Foster, S. L., Brennan, P., Biglan, A., Wang, L., \& Al-Ghaith, S. (2002). Preventing behavior problems: what Works. Recuperado de http://www. iaoed.org/files/prac08e.pdf.

Frick, P. J. (2004). The Inventory of Callous-unemotional Traits. Unpublished rating scale: University of New Orleans.

Frick, P. J., \& Marsee, M. A. (2006). Psychopathy and developmental pathways to antisocial behavior in youth. In C. J. Patrick (Eds.), Handbook of psychopathy (pp. 355-374). New York, NY: Guilford.

Frick. P. J., \& Morris, A. S. (2004). Temperament and developmental pathways to conduct problems. Journal of Clinical Child and Adolescent Psychology, 33, 54-68. doi: 10.1207/S15374424JCCP3301 6

Frick, P. J., Stickle, T. R., Dandreaux, D. M., Farrell, J. M., \& Kimonis, E. R. (2005). Callous-unemotional traits in predicting the severity and stability of conduct problems and delinquency. Journal Of Abnormal Child Psychology, 33, 471-489. doi: 10.1007/s10648-005-5728-9

Frick, P. J., \& White, S. F. (2008). Research Review: The importance of callous-unemotional traits for development models of aggressive and antisocial behavior. The Journal of Child Psychology and Psychiatry, 49(4), 359-375. doi: 10.1111/j.1469-7610.2007.01862.x

Hawes, D. J., \& Dadds, M. R. (2005). The treatment of conduct problems in children with callous-unemotional traits. Journal of Consulting and Clinical Psychology, 73, 737-741.

Huizinga D., Loeber R., \& Thornberry T. (1995). Urban Delinquency And Substance Abuse: Recent Findings From The Program Of Research On The Causes And Correlates Of Delinquency. Washington: Department Of Justice, Office Of 
Justice Programs, Office Of Juvenile Justice And Delinquency Prevention.

Kochanska. G. (1995). Children'stemperament, mothers' discipline, and security of attachment: Multiple pathways to emerging internalization. Child Development, 66, 597-615.

Kochanska, G., \& Murray K. (2000). Mother-child mutually responsive orientation and conscience development: From toddler to early school age. Child Development, 71, 417-431. doi: 10.1111/1467-8624.00154

Loeber, R., Pardini, D. Homish, L, Wei, E. H., Farrington, D. P., Creemers, J., ...Koehler, S. A. (2005). The prediction of violence and homicide in Young Men, Journal of Consulting and Clinical Psychology, 73(6) 1074-1088.

López, C. F. (2013). Inventario de Prácticas de Crianza. En C. S. Morales, \& R. M. J. Martínez (Eds.), Prevención de las Conductas adictivas a través de la atención del Comportamiento Infantil para la crianza positiva. Manual del Terapeuta (pp. 14-19). México: CENADIC-SSA.

Medina-Mora, M. E., Borges, G., Lara, C., Benjet, C., Blanco, J., Fleiz, C., ...Aguilar-Gaxiola, S. (2003). Prevalencia de trastornos mentales y uso de servicios: resultados de la encuesta nacional de epidemiología psiquiátrica en México. Salud Mental, 4, 1-16.

Morales, S. (2001). Programa de Entrenamiento Conductual a padres. En H. Ayala, C. L. Chaparro, J. M. Fulgencio, C. Pedroza, S. Morales, T. Pacheco, ...G. Mendoza, A. (Eds.), Tratamiento de agresión infantil: desarrollo y evaluación de programas de intervención conductual multi-agente. Revista Mexicana de Análisis de la Conducta, 27,1-118.

Morales, C. S. (2002). Reporte de Experiencia Profesional. (Tesis inédita de Maestría, Universidad Nacional Autónoma de México, México, D. F).

Morales, C. S. (2012). Las habilidades metodológicas y conceptuales en el quehacer cotidiano de la ciencia del comportamiento. Revista Mexicana de Investigación en Psicología, 4(2), 126-129.
Morales, C. S., \& Martínez, R. M. J. (2013). Prevención de las Conductas adictivas a través de la atención del Comportamiento Infantil para la crianza positiva. Manual del Terapeuta. México: CENADIC-SSA.

Morales, C. S., \& Santoyo, V. C. (2012). Resistencia al cambio de una conducta académica en niños. Revista Mexicana de Análisis de la Conducta, 38(1) 39-60.

Morales, C. S., \& Vázquez, P. F. (2011). Evaluación de conocimientos sobre habilidades de manejo conductual infantil en profesionales de la salud. Acta de Investigación Psicológica, 3, 428-440.

Palacios, D. J. R., \& Andrade, P. P (2008). Influencia de las prácticas parentales en las conductas problema en adolescentes. Investigación Universitaria Multidisciplinaria, 7, 7-18.

Patterson, G. R. (1982). A social learning aproach, 3, coercive family process. Eugene, OR: Castalia Publishing Company.

Scahill, L., Erenberg, G., Berlin, C. M., Budman, C., Coffey, B. J., Jankovic, J., ... Walkup, J. (2006). Contemporary assessment and pharmacotherapy of Tourette syndrome. The Journal of the American society for the Experimental Neuro Therapeutics, 3, 192-206.

Santoyo, V. C. (2012). Investigación traslacional: Una misión prospectiva para la ciencia del desarrollo y la ciencia del comportamiento. Revista Mexicana de Investigación en Psicología, 4, 2,84-110.

Speltz, M. L., Deklyen, M., Greenberg, M. T., \& Dryden, M. (1995). Clinical referral for oppositional defiant disorder: Relative significance of attachment and behavioral variables. Journal of Abnormal Child Psychology, 23, 487-507. doi: 10.1007/BF01447210

Waschbusch, D. A., Carrey, N. J., Willoughby, M. T., King, S., \& Andrade, B. F. (in press). Effects of methylphenidate and behavior modification on the social and academic behavior of children with disruptive behavior disorders: The moderating role of callous/unemotional 
traits. Journal of Clinical Child and Adolescent Psychology.

Wootton, J. M., Frick, P. J., Shelton, K. K., \& Silverthorn, P. (1997). Ineffective parenting and child- hood conduct problems: The moderating role of callous-unemotional traits. Journal of Consulting and Clinical Psychology, 65, 301-308.

Fecha de recepción: 20 de octubre de 2013

fecha de aceptación: 7 de julio de 2014 


\section{Material Suplementario}

\section{Definiciones operacionales}

Corrección simple ante el reporte escolar de mala conducta: Se refiere a la habilidad del padre/ madre ante esta situación consistente en la descripción de la conducta inapropiada del niño señalada en el reporte escolar, al indagar por los comportamientos que realizó el niño durante la escuela, en lugar de realizar la actividad académica asignada, el establecimiento de una consecuencia negativa y el establecimiento de un contrato conductual para el día siguiente.

Corrección simple a la hora de la comida: Se refiere a la habilidad del padre ante esta situación consistente en la descripción de la conducta problema y la aplicación de al menos dos consecuencias naturales (corrección simple) como por ejemplo, pedirle que limpie la mesa, que se limpie las manos, que se levante y apague la televisión, que utilice sus cubiertos o que permanezca sentado mientras come.

Corrección simple ante el berrinche: Se refiere a la habilidad del padre consistente en repetir la orden ante la que ocurrió el berrinche, ignorando la conducta inadecuada y conduciéndolo a la cama.

Corrección simple ante la obediencia a instrucciones: Se refiere a la habilidad del padre consistente en ignorar protestas, repetir la instrucción e instigar la obediencia.

Corrección simple en el supermercado: Se refiere a la habilidad del padre consistente en establecer las reglas del supermercado (por ejemplo, caminar junto a él), pedirle al niño que las repita, y la descripción de la ganancia y la pérdida por cumplimiento o no de estas.

Corrección simple a la hora de la tarea: Se refiere a la habilidad del padre consistente en describir la conducta problema, apagar la televisión y pedirle que continúe con la tarea.

Elogio. Se refiere a la habilidad del padre consistente en describir la conducta deseada, mirando, acariciando al niño y sonriéndole.

Corrección simple ante la pelea entre hermanos: Se refiere a la habilidad del padre consistente en describir a ambos niños la conducta problema, apagar la televisión y asignar actividades independientes.

Compartir: Se refiere a la habilidad del padre consistente en la comunicación verbal de cooperación o en la conducta de darle a su hijo algo que en ese momento tenga en sus manos con la finalidad de involucrarlo en la actividad. Por ejemplo, decir "tú la mitad y yo la mitad" o entregarle el balón durante un partido de fútbol para que ahora él lo utilice.

Risa provocada: Se refiere a la habilidad del padre consistente en provocar la risa en el niño, haciéndole cosquillas o contándole un chiste.

Mirar: Se refiere a mantener la mirada directa en los ojos del niño o, en su defecto, a la cara, sin importar si el niño voltea o no.

Sonreír: Consiste en levantar la comisura de los labios sin emisión alguna de sonido.

Reír: Consiste en levantar la comisura de los labios acompañado con un sonido hilarante.

Tocar: Se refiere al contacto físico que realiza el padre de forma intencional. Por ejemplo, abrazarlo.

Peticiones verbales: Son afirmaciones o comunicación verbal que hace el padre con el fin de proporcionar la oportunidad al niño de que ocurra una respuesta verbal o física en él. Por ejemplo, “y, ¿si jugamos? O ¿Por qué te gusta este juego?"

Obtener atención: se refiere a llamar al niño por su nombre, a tenerlo a un brazo de distancia y mirarlo a los ojos, antes de darle la instrucción. 
Instrucción clara: es una instrucción precisa y específica, en la cual se especifica el comportamiento que el niño debe emitir de forma afirmativa y nunca interrogativa.

Espera: es la espera de un intervalo de diez segundos para que el niño obedezca a la instrucción sin responder con protestas y quejas.

Volver a obtener atención: consiste en volver a llamar al niño por su nombre, teniéndolo cerca, a un brazo de distancia y mirarlo a los ojos, para dar la misma instrucción que no obedeció por primera vez.

Repetir literalmente la instrucción clara: se refiere a repetir la instrucción tal y como ocurrió la primera vez, de forma específica, indicando qué es lo que el niño debe hacer y solicitándolo de forma afirmativa, nunca interrogativa.

Cambiar el tono de voz: se refiere a modificar el tono de voz, dando la indicación anterior con un tono firme, no más alto ni acompañado con etiquetas, simplemente más firme que el anterior.

Esperar otros 10 segundos: consiste en volver a esperar 10 segundos a que el niño inicie la obediencia, sin responder a protestas y quejas.

Instigación física: consiste en guiar físicamente la obediencia del niño al tiempo que se repite literalmente la instrucción clara.

Instrucción clara académica: el padre obtiene atención, da instrucción clara, espera, elogia, si es necesario, vuelve a obtener atención, repite literalmente instrucción clara, cambia tono de voz, espera y, si es necesario, instiga. Por ejemplo: "Juan, haz la primera suma".

Modelar: consiste en preguntarle algún conocimiento al niño, esperar que responda, si no lo hace se le da la respuesta correcta, se le pide que la repita y se le elogia por repetir. Por ejemplo: $5+4$ son 9 , repítelo tú. ¡Muy bien! $5+4$ son 9 .

Instigar académico: consiste en preguntarle algún conocimiento al niño, esperar que responda y, si no lo hace, se le da parcialmente la respuesta, se le permite que la complete y se le elogia por completar la respuesta correcta. Por ejemplo: "5 + 4 son nue... ¡Muy bien! $5+4$ son 9".

Corregir: consiste en explicar cuál es el error que cometió el niño y pedirle que lo corrija. Por ejemplo: No, Juan, $5+4$ no son 8 , haz la suma de nuevo, por favor.

Solución de problemas: Se refiere a la habilidad consistente en describir el problema, explicar por qué es un problema, preguntar al otro por cómo se siente, ofrecer opciones de solución, evaluar ventajas y desventajas, elegir una solución y consolidar acuerdos. 